\title{
COVID-19: Key global impacts on the construction industry and proposed coping strategies
}

\author{
Ihsan Ali Husien ${ }^{1,2^{*}}$, Zelentsov Borisovich ${ }^{2}$, and Ahmed Adel Naji ${ }^{1}$ \\ ${ }^{1}$ Wasit University, Civil Eng. Department, Wasit, Iraq \\ ${ }^{2}$ Organization of construction Depart., Don State Technical University, Rostov-on-Don, 344000, \\ Russia
}

\begin{abstract}
The construction industry is an essential tributary of the global economy and an important resource in the overall product of the local economies. In December 2019, the construction industry was exposed to a shock beyond the local level as a result of COVID-19, which was classified by the World Health Organization as a global pandemic after its rapid and sudden spread in multiple countries. The nature of the effects of COVID-19 on the construction industry varied according to the diversity of its projects. The pandemic raised many challenges at the level of the workforce, low or halting productivity, production time and costs, as well as disputes in contractual formulas for construction projects. But some of the effects were more severe and caused consequences for all stakeholders in the construction sector. Researchers and those interested in the construction industry have dealt with the repercussions of COVID-19, but most of the publications focused on studying the impact of the pandemic on the local construction industry, so this paper attempts to shed light on the most prominent effects of COVID-19 on the construction industry around the world, and which were repeated with high frequency in previous studies and reports by following the methodology of review, analysis and selection, and then suggesting optimal coping strategies to reduce the damages of these effects.
\end{abstract}

\section{Introduction}

The construction industry requires the use of a set of basic resources that include local or imported raw materials, specialists and diverse manpower, financial abundance in addition to legislation and contracts that regulate the workflow. The construction industry is very diverse as it includes the construction of new buildings, the renovation and maintenance of existing buildings in addition to other types of civil engineering related projects such as roads, public utilities, transportation, energy and waste management facilities. Due to this diversity and the complex nature of the construction industry and its extension in most

* Corresponding author: ihsanali@uowasit.edu.iq 
other industries, perhaps this explains its great contribution to the global economy and its prominent role in countries, GDP. Hence and as a natural response to the boom in the global construction sector in recent years, the global demand for resources expanded dramatically and was expected to further its increase annually in the near future $[1,2]$. However, what happened at the end of 2019 after the sudden spread of the Coronavirus pandemic, or what was later called Covid-19, turned the basic concepts of the global market on its head and became one of the biggest challenges faced by humanity in the twenty-first century [3].

\section{The objective of the research}

The global community is currently striving to advise solutions and strategies to mitigate the chaos and devastating effects of COVID-19 [4]. For that this paper attempts to shed light on the most prominent impacts of this pandemic on the construction industry around the world while presenting suggested strategies to overcome these effects and reduce the current damages that is resulting from it to the construction industry. The researchers, also, hope that this study will contribute to strengthening the few available literature and expanding the field of scientific research that studies the impact of COVID 19 on the construction industry. This paper is also intended to create a scientific vision that would draw a future roadmap to face crises similar to the Coronavirus pandemic.

\section{Background}

COVID-19 was first announced at the end of December 2019 in Wuhan, China, and it soon spread very quickly in multiple regions around the world, so that prompted the World Health Organization to declare a state of emergency on March 11, 2020, regarding this disease a global health pandemic. [5]. Covid-19 has brought an unprecedented pattern of work contexts for construction companies and contractors in terms of place of work or method of communication, and has imposed clear impacts on the construction sector that must be studied and analyzed extensively [6]. During the year 2020, the researchers discussed the effects of Covid-19 on the construction industry, as the outbreak of the disease peaked, especially in Europe and the United States, and therefore this study is considered in the historical context within the category of studies of the beginning of the recovery period.

\section{Literature review}

Coronavirus pandemic will be one of the most dangerous crises in living human memory, and many expect its effects to last longer than previously thought, creating great risks around the global economy, and the construction industry is no exception [7]. Studies, articles and reports, that dealt with the effects of COVID-19 on the construction industry, have been published around the world (although they are few up to date) in response. One of the first reports is what was prepared by the Department of Business, Projects and Innovation in the Government of Ireland (August 2020) [8]. The report indicated that the construction sector was among the industrial sectors most affected by the Coronavirus, as 
the activity shrank by about $50 \%$ and according to the European Central Bank's economic forecasts, the construction sector will suffer a loss of about $40 \%$.

Borkova et al. (2020) [9] in a study on the digital transformation of the construction industry in the context of the macroeconomic shock as a result of COVID-19, they indicated that during the intensity of the quarantine period, about 58 construction establishments across Moscow were closed and they concluded that the use of digitization in the construction industry would reduce the effects of the Coronavirus pandemic. Jachhoun (2020) [1] hinted in his study on assessing the impact of Covid-19 on the construction industry in Ethiopia that if the epidemic continues for 6 months, there would be about 1.76 million jobs at risk. In a study prepared by the National University of the Maldives (August 2020) [10] about the impact of COVID-19 on the construction industry in the Maldives (preliminary assessment) the research team cited the data of the Contractors Association, which indicated that the effects of COVID-19 in the Maldives are similar to its effects on the construction industry around the world and these impacts included disruption or stopping construction projects, financial problems, increased costs, workforce problems, supply chain disruptions, as well as legal problems related to contract terms.

Ogunnusi et al. (November 2020) [11] in their study regarding the Coronavirus pandemic, implications and expectations, and after reviewing the literature and surveying construction industry professionals from 15 countries in five continents, indicated that some construction companies may resort to borrowing for fear of bankruptcy as a result of the effects of COVID-19 also they concluded that construction experts may rely more on modern technologies for the purpose of increasing production. Bseisu (2020) [12] studied, in his research, the effects of COVID-19 on Jordanian civil engineers, whether working in offices or on the work site, in the construction industry. He found that, throughout a questionnaire, $47.1 \%$ of office engineers did not notice a change in their productivity, while $31.8 \%$ indicated a lack of productivity, and $21.2 \%$ noticed an increase in their production, and $39.3 \%$ believed that some engineers may lose their jobs due to the effects of the COVID-19 pandemic.

\section{Methodology}

This study relied on collecting the limited information available from various sources including previous literature, government reports, and electronic publications published by construction organizations or companies around the world. The researchers attempted to study and analyze all the information contained in the publications of construction specialists about the types of impacts of COVID-19 on the local construction industry and then shed light on the most prominent of these influences, which had a global impact, and suggest strategies to reduce damages commensurate with the nature of these effects.

\section{Impact of COVID-19 on the global construction industry}

Covid-19 not only affected human health, but its effects extended to include almost all aspects of life: health, social and economic. As a result, the global economy deteriorated to its worst level in decades and the construction sector in Russia, America, Europe and other 
countries suffered various consequences such as suspending or canceling activities, lack of suppliers' ability to deliver essential construction materials, uncertainty regarding future projects, workforce problems, increased costs and expiration the time in addition to a set of legal issues [13]. The following is a statement of the most prominent effects of Covid-19 on the construction industry.

\subsection{Problems related to Workforce}

Based on the report of the International Labor Organization, approximately 2.7 billion workers, equivalent to $81 \%$ of the workforce around the world, would be affected by COVID-19, and certainly these percentages include workers in the construction sector. Where the researchers pointed in an Omani study to the significant decline in employment after the entry of COVID-19, and they attributed this decrease to the increase in mortality and social distancing [4]. A survey also indicated, through interviews with 13 construction professionals from various disciplines in 10 British companies, that $46 \%$ of them work temporarily remotely, $15 \%$ are still working on the site, and $16 \%$ have lost their jobs [6]. The situation did not differ much in Nepal, where the number of workers, whether local or expatriate, before the pandemic was 1.7 million, while reports indicated that there are only 300,000 workers, or $5 \%$, are left and they earn about $50 \%$ less than their previous wages [14].

China is largely dependent on rural migrant workers, bout 54 million workers. As a result of lockdown policies, a large part of them were unable to return to work sites after the Lunar New Year holiday in 2020 [15]. The workforce has also suffered from great psychological burdens as a result of worrying about their future, and the financial difficulties that they may face them, especially the majority of them have financial obligations and families [5]. This is what prompted many workers in the informal construction sector to continue working despite the epidemic, exposing themselves and their families at risk [2].

\subsection{Global supply chain disruption}

The extent of the COVID-19 shock was global rather than local which disrupted most supply chains around the world and reduced or halted most international commercial activities related to the supply of construction materials, causing major delays in the projects [16]. The International Labor Organization indicated that the Covid-19 crisis has caused disruption to most of the materials supply transactions, and equipment rental companies have complained of major problems due to the remaining defective equipment at the sites [2]. In addition to the reports of contractors' associations in the Pacific Ocean, including the Indian, Japanese and Korean Construction Association, etc., which clearly showed that the supply chain in their countries has been affected by COVID-19 to encounter a severe shortage of imported construction materials in most local markets. This was also recorded in the markets of the Maldives after imports from China or India have ceased [10]. In Ethiopia, there has been a great disruption in steel, aluminum and cement supplies, as countries that export these materials, such as China, the United States or the United Kingdom implemented a policy of closure [1]. It should be noted that the construction industry in Australia in the past ten years has relied heavily on importing 
construction materials such as aluminum, plumbing fixtures, glass, etc., especially from China. Therefore, it was observed that there was a significant effect at the beginning of the pandemic on construction projects in Australia, especially on large projects. Whereas the effect was lower on small projects, since they rely more on local raw materials [17]. In addition, in a survey conducted by Suiko (as part of Turner \& Townsend) on 45 projects completed during the Coronavirus pandemic, about $7 \%$ of productivity losses were due to lack of materials [11]. The disruption in the supply chain may not only be global but also at the local level, as it happened in the United States, where due to the increasing concerns of truck drivers of COVID-19 (or the consequences of a 14-day quarantine) when crossing the state borders. Many of them hesitated to transport materials and consequently there was a scarcity of materials on construction sites [5].

\subsection{Legal issues}

Legal disputes and claims have significantly affected the local construction industry around the world. Researches and reports related to the construction industry indicated that there is a controversy over the legal interpretation of the impact of the Coronavirus pandemic on construction projects. Some specialists believe that it is possible to interpret COVID-19 as being of the category Force Majeure (unforeseeable circumstances in which contractors are unable to fulfill a contract) while others tend to the principle of changing laws to resolve disputes. Interpretation of the pandemic as a Force Majeure, as it is found in most contracts including FIDIC, allow the contractors to claim compensation for the time resulting from the delay, but it does not give them the right to claim financial compensation except in case of agreement between the parties.

In contrast to Force Majeure, the scenario of changing laws will allow the contractor extending the time and claim financial compensation due to legal changes. Most international contracts such as NEC and JCT use protocols related to the issue of Force Majeure, but there is no unified definition for it [18], and most of the local laws and contracts for construction projects in the world have included references to explain Force Majeure. For example, the 1960 Civil Code stipulates in Article 1793. In Ethiopia, Force Majeure is an unexpected external event, where both parties to the contract are not responsible for it, such as if it is a security event specific to the state or a natural disaster [1]. At the time when construction contracts in Amman faced difficulties in finding legal solutions to disputes in construction projects during the pandemic because the state's legislation does not give a legal interpretation of the Force Majeure principle [4].

Legislation in China was flexible during the outbreak of the epidemic, as the Standing Committee of the National People's Congress indicated that COVID-19 is a sudden and unexpected event and therefore the contracting parties can exempt from the implementation of their obligations [15]. In the questionnaire about the impact of COVID-19 on the civil engineers in Jordan, only $40.7 \%$ of engineers were aware of the existence of a Force Majeure clause in the legal contract regulations, but interpreting COVID-19 as a Force Majeure would be based on the nature of the approved contracts [12].

The legal effects of COVID-19 were not limited to construction contracts only, but extended to include relations between the participants in the projects, so there were disputes between stakeholders and contractors due to delay or lack of completion, other disputes 
between contractors and subcontractors, as subcontractors may demand a new mechanism of pricing for the project activities. Also disputes arose in the relationship between the contractor and the insurance companies as to whether the insurance policies cover the pandemic issue or not [5]. There is no doubt that construction companies and contractors faced many other legal complications, such as the percentage of profits, increased costs, new safety conditions and the issue of taxes, etc. This requires difficult negotiations, given that the contractual terms are not commensurate with price fluctuations or the stoppage of project activities as a result of epidemics. Thus, these legal disruptions have motivated many contractors to activate contractual formulas that allows them to work in more flexible legal conditions $[2,14,19]$.

\section{Coping strategies to reduce damages}

After highlighting the most prominent impacts of COVID-19 on the construction industry, which has tangibly and globally affected this sector, visions should be unified to develop effective strategies with realistic and applicable protocols that contribute to mitigating the current and continuing effects of the pandemic. It is also possible to adopt these strategies in the future to face the global crises as the Coronavirus pandemic [20]. Possible and effective strategies can be summarized below.

\subsection{Safe work site conditions}

It can be said that the project workforce, with its skilled and unskilled laborers, is the core of the construction industry, so all necessary measures should be taken and all available capabilities harnessed in order to protect this resource from exposure to the risk of COVID19 and its dangerous health consequences [20]. This forces all key players in the implementation of projects, whether construction organizations or contractors, to develop new occupational safety standards and protocols to face the critical health challenges which are results of COVID-19. Thus, it will maximize the safety factor for construction workers at the site [1]. Construction management can achieve this factor by adopting strict health policies, including ensuring a safety distance between workers and the use of protective masks (despite the difficulty in achieving these two conditions during work), urging workers to physical hygiene and the use of disinfectants, good ventilation, as well as improving health care [20].

Achieving the requirements of the safe work site conditions requires the stakeholders to manage the services inside the site in an innovative method, where it is possible to place warning signs reminding workers about the issue of physical spacing, not allowing a food truck to enter inside the site to prevent congestion, closing the dining hall and eating in open places. In addition, it may require to reduce the number of the workers present on the site by following the work shifts at different times and directing the non-essential workers to work at home with the possibility that supervisors work on site from their cars to avoid contact with the rest of the workers [5].

Mental health professionals should be available on the construction site as increased worker anxiety about the risk of contracting COVID-19 will cause confusion in the chain of command and directions and then decrease in productivity [6]. Some construction 
companies and organizations have taken the first steps of a damages reduction strategy in the United States, as an example Truebeck company has adopted a preventive system based on checking the temperature of workers, providing personal protection supplies and placement of health signs in the site. San Mateo County also requested a safety specialist who conducts random interviews with site workers through the online-meeting app (Zoom for instance) to verify the reliability of their health conditions at work. While, Hanover company has contracted nurses to measure workers' temperatures at workplace entrances [21].

\subsection{Creative and innovative solutions}

The construction industry should go more towards automating processes and leaving the traditional methods of implementation [3]. To achieve this strategy, hypothetical alternatives can play an effective role, as correspondence and meetings can take place through electronic applications [11]. Also, the project manager can manage claims and communicate with contractors via video calls [19] or through an interactive electronic platform for direct communication among all stakeholders [22]. BIM and its distinct methodology in resource management can be used to proactively anticipate building materials required in project activities [9]. Contractors, on their part, have the task of early monitoring of the global supply chain [7] and continuous examination of the schedule for the project activities. They also have to search for reliable alternatives from local suppliers of construction materials [5].

Another type of tactics is through diversifying supply chains and avoiding being satisfied with a single source of supply [23] or by adopting decentralization by establishing a network of supply centers from easily accessible areas [3]. Construction organizations should have a bold, creative vision to look at the technological options available during the pandemic period. These options vary from pre-fabricating off-site [6] or using laser scanners to measure production, drones to monitor the site, or even using robots or 3D printing in construction processes. These Techniques help to reduce the human factor on work sites [9].

\subsection{Cooperative and fair legislation}

There is a need for all parties to negotiate in a cooperative legal approach that brings justice to bear the consequences arising from the impact of COVID-19 and ensures the continuation of activities. Contracts must be reviewed and amendments may be made to both local regulations and standard contracts to settle disputes over time extension and financial obligations [18]. There is a real need to review standard contracts, including FIDIC, as global pandemics must be included as Force Majeure in construction contracts [23]. It is not correct to deal with contracts as individual cases rather, dealing must be within a comprehensive vision of the magnitude of the crisis in which the rights and obligations of contractors or project owners are determined [14].

Countries and governments should take the initiative through steps that contribute to minimizing damages, such as providing Covid-19 tests for contractors to check workers, especially foreigners [13], amending occupational health and safety regulations in contracts [11], put fair tax policies that allow local suppliers to compete in the material market [3], 
providing bank lending facilities with a low interest rate, especially for small companies [2]. Also, customers contracting with construction companies must tolerate the issue of delay resulting from COVID-19 [7]. Contractors must provide all the reasons for the delay and increase in costs, which will be useful in developing balanced contractual formulas [5]. Finally, contractors had global projects should seek advice from a consultant expert in international laws to propose a negotiation strategy that is compatible with the global legal challenges posed by the Coronavirus pandemic [24].

\section{Conclusion}

It is evident from the information available in previous studies and publications that the construction industry is a vital artery in providing jobs in several countries and therefore millions of the workforce have suffered from financial crises or psychological problems as a result of this sector being affected by COVID-19, which has caused huge disturbances affecting the various construction industry resources and its legal legislations. Many countries did not consider the construction industry an essential part during the outbreak of the pandemic, unlike some sectors such as health, education and the provision of necessary supplies, and consequently the construction industry suffered remarkably from the closure policies practiced by the world's governments, whether the policy of quarantine or the closure of international borders consequently, most supply chains have been disrupted and inability of suppliers to deliver key construction materials for projects, especially large and complex ones, which caused most of the activities to stop and delayed completion, also additional financial and administrative consequences that affected some of the parties participating in the project more than other parties and thus generated an increase in claims for compensation or may be due to the nature of standard contracts or contractual formulas Local practice in the construction industry, which was supposed to be more accurate in describing the legal interpretation of global crises such as epidemics. The researchers concluded that the effects of COVID-19 were wide and varied, but the most important of them were problems related to the workforce, disruption of supply chains and legal disputes, and other problems and impacts may be reflections of the three effects that have been mentioned. Researchers believe that the Corona pandemic may be an opportunity to change traditional policies in management. Projects and moving more towards modernity and automation, taking advantage of some modern technologies and computer applications, with more attention to the issue of occupational safety and the safety factor of the workforce, especially in developing countries, and the possibility of reformulating contracts and legal legislations in a way that achieves the principle of justice for all shareholders, and this is what researchers tried to explain in the proposal of coping strategies to reduce damage. In conclusion, it should be noted that this study has suffered from a scarcity of information due to the dearth of literature on the effects of COVID-19 on the construction industry.

\section{Reference}

1. A. D. Gashahun, IJESC, 10, 7 (2020)

2. ILO, Sectoral Brief (2020) 
3. L.P.D.S. Pathirana, SSRG Int. J of Eco. and Manage. Stu., 7, 6 (2020)

4. T. Al Amri, M. Marey-Pérez, Tech. Soc. Scie. J, 9 (2020)

5. A. Alsharef, S. Banerjee, S. M. Jamil Uddin, A. Albert, E. JaselskisInt, J. Environ. Res. Public Hea, 18, 1558 (2021)

6. M. Stride, S. Suresh, S. Renukappa, J. Environ. Res. Public Hea, (to be published)

7. Mace, Man. Imp. and R. on Cons. Ind. J (2020)

8. Government of Ireland, Foc. on Con., August (2020)

9. E.A. Borkova, A.G. Izotova, N.A. Litvinova, Issues of Innovation Economy, 10, 4 (2020)

10. The Maldives National University (2020)

11. M. Ogunnusi, M. Hamma-adama, H. Salman, T. Kouider, Int. J of R. Est. Stu., 14, S2 (2020)

12. K. A. Bsisu, Int. J of Eng. Res. and Tech., 13, 5 (2020)

13. F. S. Ibrahim, Adv. in Sci. Tech. and Eng. Sys. J, 5 , 5 (2020)

14. The Asia Foundation, (2020)

15. FTI Consulting, Inc., (2020)

16. C. Bond, D. Quan, B. Goldberg, V. Calanog, REIS Re. Est. Sol. by Moo. Ana. J (2020)

17. T. Emmett, https://www.turnerandtownsend.com/en/perspectives/covid-19-and-theimpact-on-australian-construction-projects.

18. A. E. Yadeta, D. Pandey, Curr. Tren. in Civ. \& Stru. Eng. J, 6, 4 (2020).

19. Y. Gamil, A. Alhagar, Medit. J of Soc.Scie., 11, 4 (2020)

20. V. Kaushal, M. Najafi, Civ. Eng. Bey. Lim. J, 2, 8 (2021)

21. E. Bohannon, Cal Polytec. St. Uni. J (to be published)

22. S. Nahidi, N. Ibrahim, S. Tighe, (to be published)

23. RICON FOCUS, 3 (2020)

24. Jones Day White Paper, (2020) 